\title{
Limitations of T2*-Gradient Recalled-Echo and Susceptibility- Weighted Imaging in Characterizing Chronic Subdural Hemorrhage in Infant Survivors of Abusive Head Trauma
}

\author{
(D).A. Cramer, (D).A. Rassner, and DG.L. Hedlund
}

\begin{abstract}
SUMMARY: A possible misconception among radiologists is that chronic subdural hemorrhage should show some degree of blooming on T2*-gradient recalled-echo or susceptibility-weighted sequences such as SWI and susceptibility-weighted angiography, which is not necessarily true. We present 5 cases of chronic subdural hemorrhages in infants, demonstrating intensity near or greater than that of CSF with variable amounts of hemosiderin staining along the neomembranes. We review the physiology and MR imaging physics behind the appearance of a chronic subdural hemorrhage, highlighting that the absence of a BBB can allow hemosiderin to be completely removed from the subdural compartment. Finally, we stress the importance of reviewing all multiplanar sequences for the presence of neomembranes, which can be quite subtle in the absence of hemosiderin staining and are critical for making the diagnosis of chronic subdural hemorrhage.
\end{abstract}

ABBREVIATIONS: GRE = gradient recalled-echo; $\mathrm{CSDH}=$ chronic subdural hemorrhage; $\mathrm{SDH}=$ subdural hemorrhage; $\mathrm{SDHy}=$ subdural hygroma; $\mathrm{SWAN}=$ susceptibility-weighted angiography

M uch of the literature characterizing the MR imaging appearance of subdural hemorrhage (SDH) refers to T1 and T2 signal, with no explicit discussion of the appearance on $\mathrm{T} 2{ }^{*}-$ gradient recalled-echo (GRE) or susceptibility-weighted sequences such as SWI or susceptibility-weighted angiography (SWAN). Moreover, one may reason that a chronic subdural hemorrhage (cSDH) should show blooming or hypointensity on T2*-GRE or SWI, but this is not necessarily true. ${ }^{1-3}$ We present 5 infant survivors of abusive head trauma with subdural collections demonstrating intensity near that of CSF on $\mathrm{T} 2{ }^{\star}$-GRE or hyperintensity on SWAN with variable amounts of hemosiderin staining. These cases either exhibited other imaging features compatible with cSDH or were called cSDH on surgical drainage. We provide a review of the physics and physiology relevant to the appearance of chronic SDHs on MR imaging and offer an explanation for the subdural fluid signal and variable hemosiderin staining on $\mathrm{T} 2^{\star}$ GRE and SWI.

Received November 16, 2015; accepted after revision February 8, 2016.

From the Department of Radiology (J.A.C., U.A.R.), University of Utah Hospital, Salt Lake City, Utah; and Department of Radiology (G.L.H.), Primary Children's Hospital, Salt Lake City, Utah.

Paper previously presented at: Annual Meeting of the American Society of Neuroradiology and the Foundation of the ASNR Symposium, April 25-30, 2015; Chicago, Illinois.

Please address correspondence to Justin A. Cramer, MD, University of Utah Radiology, 30 N 1900 E \#1A71, Salt Lake City, UT 84132; e-mail: jcramer10@gmail.com; @justincramer

http://dx.doi.org/10.3174/ajnr.A4769

\section{CASE SERIES}

Illustrative cases were obtained by reviewing neurosurgical patients in whom drainage of a subdural collection was performed at Primary Children's Hospital between 2009 and 2014. We selected infants (younger than 12 months of age) with an operative report indicating chronic subdural hematoma and MR imaging $<1$ week before surgical drainage. Those with acute or chronic subdural hematomas were excluded. One hundred fifty-six total subdural drainage procedures were performed during our study period, and 5 patients were selected. A large majority of cases were not used due to acute hemorrhage, subdural hygromas, or lack of MR imaging within 1 week of drainage. In all 5 selected patients, a diagnosis of abusive head trauma was confirmed by the institutional child protective services team. Operative description and pathology were recorded when available. Results are summarized in the Table.

\section{Case 1}

A 4-month-old boy presented with increasing head circumference and no outward signs of trauma. Bilateral subdural hematomas were demonstrated on NCCT. Findings of 2 osseous surveys were negative, but subsequent investigation led to the diagnosis of inflicted trauma/abusive head trauma. NCCT and MR imaging (Fig 1) were performed 4 and 3 days before surgical drainage, respectively. NCCT demonstrated bilateral subdural collections that were hyperattenuating to CSF. On MR imaging, the subdural fluid was hyperintense to CSF on FLAIR and slightly hyperintense 
Case series of infants with CSF-like subdural collections on T2*-GRE/SWI

\begin{tabular}{|c|c|c|c|c|c|}
\hline Case & Age (mo) & Sex & MRI Diagnosis & Operative Description & Pathology (If Available) \\
\hline 1 & 4 & $M$ & $\begin{array}{l}\mathrm{cSDH}(\uparrow \mathrm{T} 1 / \mathrm{T} 2 / \mathrm{FLAIR} / \mathrm{SWAN}, \\
\text { hemosiderin along neomembranes) }\end{array}$ & "Subdural hematoma" & Culture, no growth; no cell count \\
\hline 2 & 5 & M & $\begin{array}{c}\text { cSDH ( } \uparrow \mathrm{Tl} / \text { FLAIR/SWAN/GRE, iso T2, } \\
\text { hemosiderin along neomembranes) }\end{array}$ & "Reddish-brown CSF" & Culture, no growth; no cell count \\
\hline 3 & 6 & M & SDHy (iso to CSF, no neomembranes) & $\begin{array}{l}\text { "Chronic subdural } \\
\text { hematoma" }\end{array}$ & Not sent \\
\hline 4 & 4 & M & $\begin{array}{l}\text { cSDH ( } \uparrow \text { T1/FLAIR, } \downarrow \text { GRE/T2, } 1 \\
\text { neomembrane with no hemosiderin) }\end{array}$ & $\begin{array}{l}\text { "Blood-tinged hematoma } \\
\text { fluid" }\end{array}$ & $\begin{array}{l}\text { Culture, no growth; WBC } 945 / \mu \mathrm{L}(\mathrm{H}) \text {; } \\
\text { RBC } 149,188 / \mu \mathrm{L}(\mathrm{H})\end{array}$ \\
\hline 5 & 4 & M & $\begin{array}{l}\text { CSDH ( } \uparrow \text { TI/FLAIR/GRE, iso T2, } \\
\text { hemosiderin along neomembranes) }\end{array}$ & "Blood-tinged CSF" & Culture, no growth; no cell count \\
\hline
\end{tabular}

Note:- $\uparrow$ indicates increased signal; $\downarrow$, decreased signal; iso, isointense; WBC, white blood cell count; RBC, red blood cell count; $H$, high.

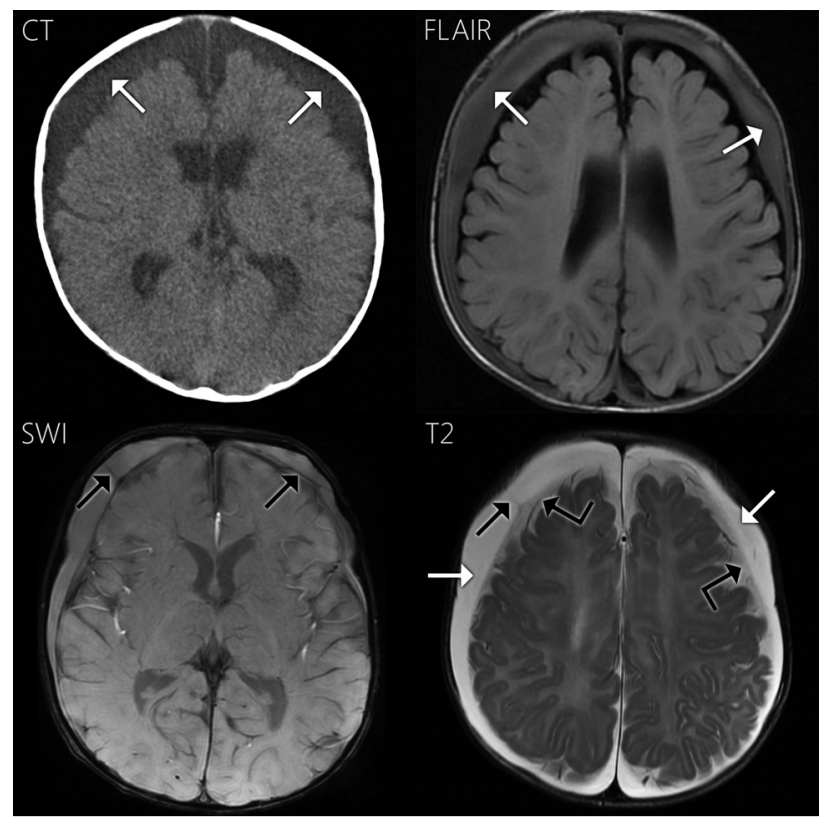

FIG 1. Case 1. Axial NCCT demonstrates bilateral subdural collections, slightly hyperattenuating to CSF (white arrows). The collections are hyperintense to CSF on FLAIR and more subtly hyperintense on TIWI (not shown). On SWAN, the fluid is hyperintense to CSF. Neomembranes with hemosiderin staining are present bilaterally within the subdural compartment on SWAN and T2WI (black arrows). Hemosiderin staining along the right-sided neomembrane shown is quite subtle. On T2WI, the subdural fluid is hyperintense to the adjacent subarachnoid CSF (angled black arrows) and isointense to ventricular CSF (not shown).

on T1WI. On SWAN, subdural compartment neomembranes with hemosiderin staining were seen bilaterally, compatible with cSDH. The subdural fluid was hyperintense to CSF on SWAN. T2WI also demonstrates subdural compartment neomembranes. On T2WI, the subdural collections (white arrows in Fig 1) were hyperintense to the adjacent subarachnoid fluid (angled black arrows) and isointense to ventricular CSF (not shown). The neomembranes did not enhance. The operative report described the fluid as "subdural hematoma."

\section{Case 2}

A 5-month-old boy was brought in for vomiting and was found to be somnolent. NCCT showed subdural compartment collections prompting an inflicted trauma evaluation, which revealed a healing left humerus fracture. NCCT was performed 1 day before surgery, and MR imaging was performed the day of surgical drainage (Fig 2). NCCT demonstrated slightly hyperattenuating

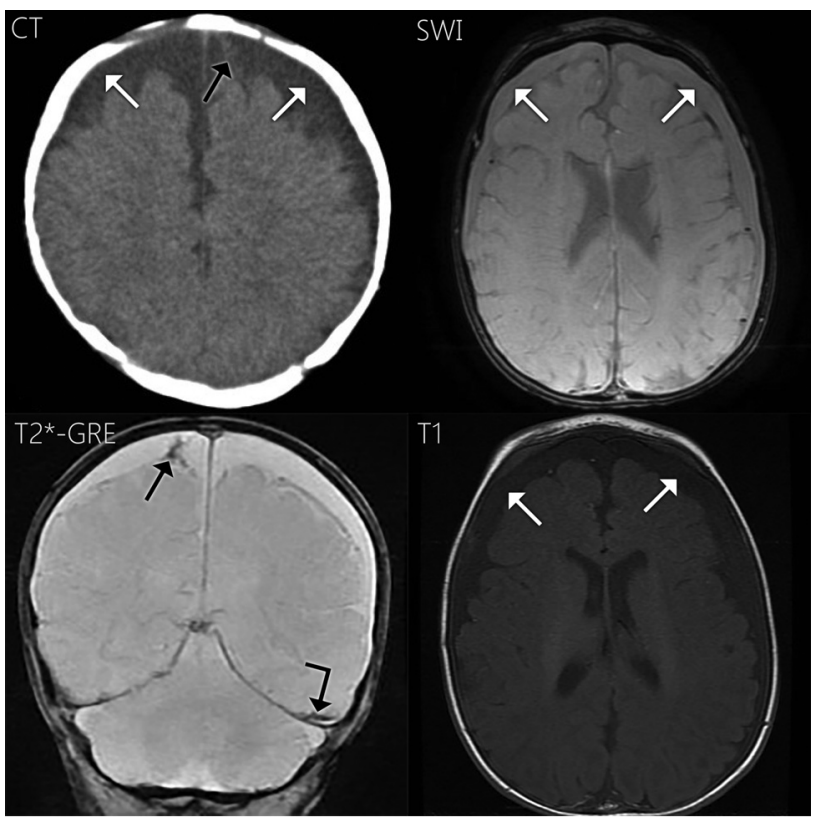

FIG 2. Case 2. Axial NCCT shows bilateral subdural collections slightly hyperattenuating to CSF (white arrows) and hyperattenuation along a neomembrane within the medial left frontal subdural collection (black arrow). The subdural fluid is slightly hyperintense to CSF on FLAIR (not shown) and TIWI (white arrows). On T2*-GRE, the subdural fluid is slightly hyperintense to CSF with blooming along a subdural compartment neomembrane (black arrow) and the left tentorium (angled black arrow). On SWAN, the subdural fluid is hyperattenuating to CSF (white arrows). Hemosiderin staining was less conspicuous in the axial plane on SWAN.

bilateral subdural collections and a left frontal subdural compartment neomembrane. On MR imaging, the subdural fluid was slightly hyperintense to CSF on FLAIR and T1WI. The subdural fluid was slightly hyperintense to CSF on T2*-GRE. Blooming was noted along a subdural compartment neomembrane and along the left tentorium. Contrast was administered with no enhancement along the membranes observed. The subdural fluid was hyperintense to CSF on SWAN, and neomembranes were less conspicuous on SWAN in the axial plane. On T2WI, the subdural fluid was isointense to CSF and the neomembranes were also seen. The neomembranes were compatible with cSDHs. The operative report described the fluid as "reddish-brown CSF."

\section{Case 3}

This 6-month-old boy was a survivor of witnessed inflicted injury. Skeletal survey revealed numerous healing fractures. NCCT 


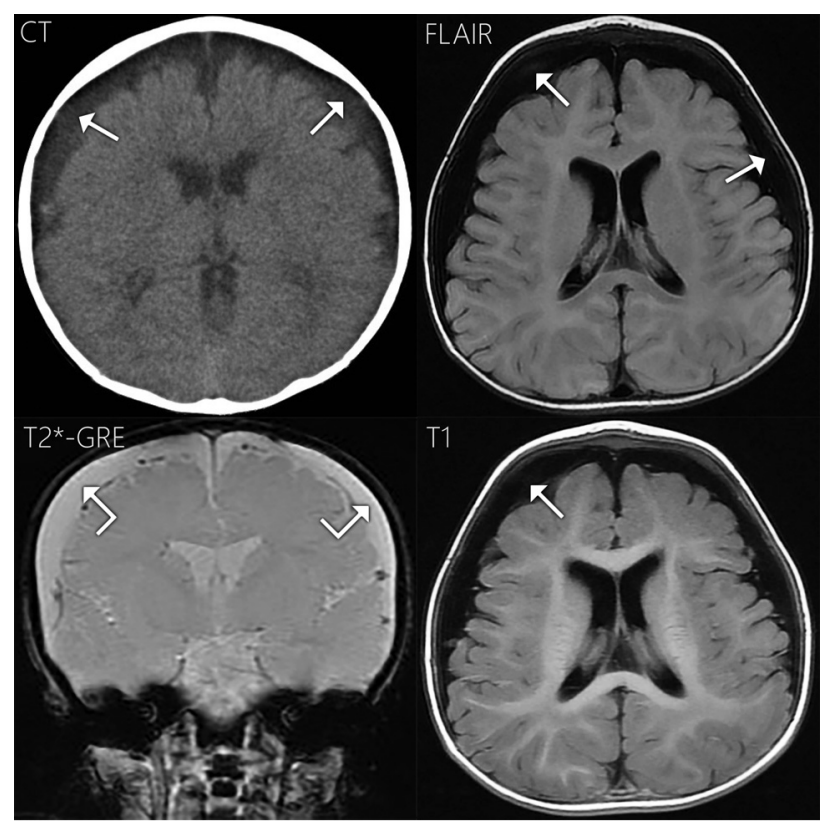

FIG 3. Case 3. Axial NCCT demonstrates bilateral subdural collections slightly hyperattenuating to CSF (white arrows). On FLAIR and TIWI, the subdural fluid is nearly isointense to CSF with subtle areas of hyperintensity reflecting CSF flow artifacts (white arrows). On T2*GRE, the subdural fluid is isointense to CSF (angled white arrows) with no blooming or susceptibility effects along the dural surface. The multiple tubular hypointense structures on T2*-GRE are cortical veins.

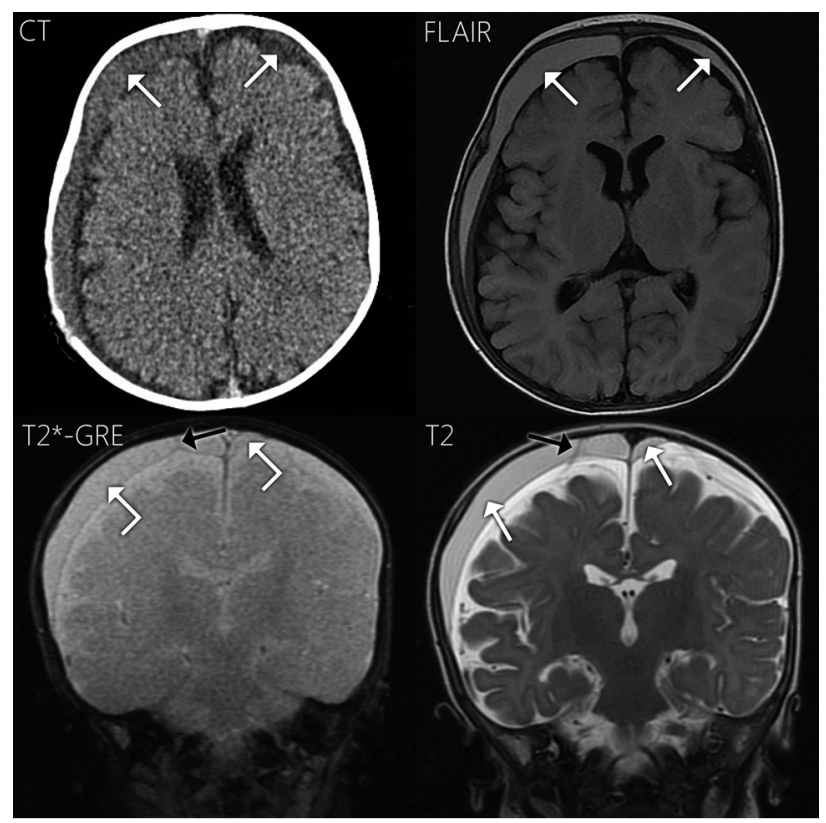

FIG 4. Case 4. Axial NCCT shows right-larger-than-left subdural hematomas hyperattenuating to CSF (white arrows). On MR imaging, the collections are hyperintense to CSF on TIWI and FLAIR (white arrows). The collections approximate the signal of CSF on T2*-GRE (angled white arrows) and are notably hypointense on T2WI (white arrows). Note the right frontal subdural compartment neomembrane on T2*-GRE (black arrow), with very subtle hemosiderin staining, slightly more conspicuous on T2WI (black arrow).

(Fig 3) showed bilateral subdural collections that were isoattenuating to CSF. The fluid was predominantly isointense to CSF on FLAIR and T1WI, with subtle areas of hyperintensity. The fluid

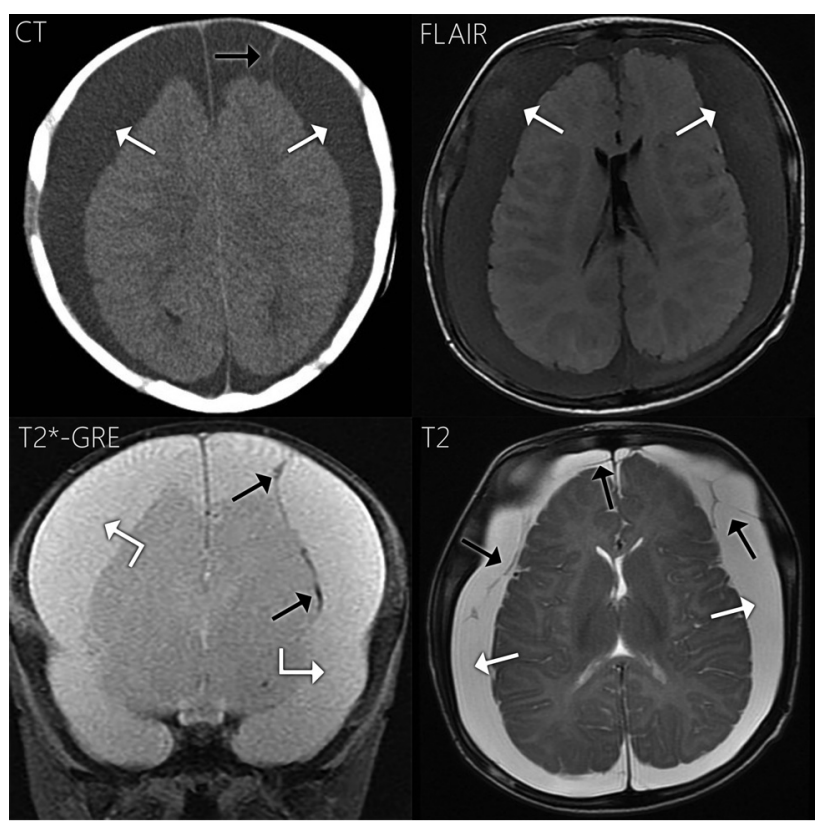

FIG 5. Case 5. Axial NCCT demonstrates large bilateral subdural collections hyperattenuating to CSF (white arrows) and a hyperattenuating subdural compartment neomembrane (black arrow). On TIWI (not shown) and FLAIR, the subdural fluid is hyperintense to CSF (white arrows). On T2*-GRE, the subdural fluid is slightly hyperintense to CSF (angled white arrows), with hemosiderin staining along a leftsided neomembrane (black arrows). On T2WI, the subdural fluid is isointense to CSF (white arrows) and nicely demonstrates multiple neomembranes (black arrows).

was isointense to CSF on T2*-GRE and T2WI with no apparent neomembranes or hemosiderin staining (Fig 3). Contrast was not administered. The collections were interpreted as hygromas on the basis of the imaging appearance. The operative report 3 days later described the fluid as "chronic subdural hematoma."

\section{Case 4}

This 4-month-old boy was evaluated for increasing head circumference and was subsequently found to have bilateral subdural hematomas on NCCT (Fig 4). Child protective services evaluation raised concern for inflicted injury. On MR imaging (Fig 4), the subdural collections were hyperintense to CSF on FLAIR and T1WI and slightly hypointense to CSF on T2*-GRE. Hypointensity of the collections was more pronounced on T2WI. A single subdural compartment neomembrane was also observed on $\mathrm{T} 2{ }^{\star}$ GRE with no hemosiderin staining. This membrane was slightly more conspicuous on T2WI. Contrast was not administered. The collections were called cSDHs based on the MR imaging appearance. Surgical drainage the next day resulted in a description of the subdural fluid as "blood-tinged hematoma fluid."

\section{Case 5}

This 4-month-old boy presented with increasing head circumference and vomiting. NCCT (Fig 5) revealed bilateral subdural hematomas with internal neomembranes. In addition, there were bilateral parietal bone fractures. On MR imaging (Fig 5), the subdural fluid was hyperintense to CSF on FLAIR and T1WI. On T2*-GRE, the fluid was slightly hyperintense to CSF and demonstrated internal neomembranes with hemosiderin staining along 
the neomembranes. The membranes were also quite conspicuous on T2WI. Contrast was not administered. Surgical drainage 2 days later revealed "blood-tinged CSF."

\section{DISCUSSION}

We present 5 infants with chronic posttraumatic subdural hemorrhage in which the signal of the subdural fluid was near that of CSF on T2*-GRE or hyperintense on SWAN. Four cases demonstrated subdural compartment neomembranes, with very subtle or absent hemosiderin staining along the neomembranes in 2 cases. These observations of subdural fluid signal near or greater than that of CSF and subtle or absent hemosiderin staining may be somewhat counterintuitive because one may reason that chronic subdural hemorrhage should exhibit hypointensity due to susceptibility effects. However, this supposition is not necessarily true and raises several important points.

Wittschieber et $\mathrm{al}^{4}$ posited that most acute SDH resolves completely and only rarely goes on to form cSDH. More commonly, a subdural hygroma (SDHy) serves as an intermediary step between acute $\mathrm{SDH}$ and cSDH. Essential to the formation of a $\mathrm{CSDH}$ is the development of vascularized subdural compartment neomembranes. These neomembranes are prone to effusing CSF and microhemorrhages. ${ }^{4}$ Therefore, a cSDH is a mixture of blood-breakdown products, CSF, and neomembranes.

How do these components of a cSDH contribute to MR imaging signal? First, consider the evolution of subdural blood. It goes through the same hyperacute, acute, early subacute, and late subacute stages as parenchymal blood, just more slowly. This slower progression has been attributed to greater oxygen tension in the subdural compartment. ${ }^{2,5}$ However, the chronic stage of subdural hemorrhage differs from that in parenchymal hemorrhage. A $\mathrm{cSDH}$ is characterized by repeat episodes of rebleeding along vascularized neomembranes, thus containing varying amounts of albumin, hemoglobin, and hemoglobin breakdown products, including hemosiderin and globin proteins. ${ }^{6,7}$ The various proteins increase T1 and FLAIR signal, and paramagnetic/ferromagnetic hemosiderin causes blooming on T2*-GRE/SWI. Hemosiderin tends to layer along the dural surface and along neomembranes. However, hemosiderin may be completely absent from the subdural compartment ${ }^{1-3}$ because absence of a BBB allows hemosiderin-laden macrophages to scavenge blood products and exit the subdural compartment. ${ }^{2}$ Contrast this scenario with chronic parenchymal hemorrhage, which typically shows hemosiderin staining. This explains the range of $\mathrm{cSDH}$ signal in our 5 cases: variable T1/FLAIR hyperintensity to CSF due to proteins and variable hemosiderin staining on T2*-GRE/SWI. Several of our cases demonstrated absent or very subtle hemosiderin staining along the neomembranes. Finally, the fluid in chronic subdural hemorrhage does not need to be hypointense on T2*-GRE/SWI. ${ }^{3}$ In our cases using SWAN, the fluid was hyperintense to CSF. On T2*-GRE, 2 of our cases demonstrated slight hyperintensity relative to CSF: One demonstrated slight hypointensity, and another demonstrated isointensity. The hyperintensity relates to the same proteinaceous content that elevates the T1 and FLAIR signal, and the slight hypointensity reflects the susceptibility effects of blood products.

Neomembranes are important to note because they are diag- nostic of cSDH and, along with non-CSF-like subdural fluid signal, represent the key observations allowing differentiation from SDHy. However, they are of varying conspicuity, with one of our cases demonstrating no definite neomembranes. T2*-GRE/SWI can make neomembranes more conspicuous, particularly in the presence of hemosiderin staining. Additionally, neomembranes can enhance, and contrasted imaging may improve membrane conspicuity in some authors' experience. ${ }^{5,8}$ Of note, intravenous contrast provided no additional information in our 3 contrastenhanced cases. Finally, membranes may be much more conspicuous in a particular plane, which highlights the importance of multiplanar imaging. If no membranes are identified and there is no hemosiderin staining, differentiating among hyperacute SDH, $\mathrm{SDHy}$, and cSDH can be difficult. In such challenging cases, complementary nonenhanced CT and serial imaging are useful because these collections will evolve differently. ${ }^{9}$

Variable detail in the operative reports limits our correlation of imaging appearance with surgical drainage findings. cSDH fluid has often been described as having the gross appearance of "crank case oil" - that is, thick and dark red/brown. ${ }^{4,6,10}$ Most interesting, cases 4 and 5 had operative descriptions more typical of SDHy, yet they still had membranes compatible with cSDH. This further highlights the complex and varying nature of cSDH fluid. Case 3 had the imaging appearance of an SDHy, yet it was called "chronic subdural hematoma" in the operative report. Given the retrospective nature of this report, it is unclear how to reconcile this discrepancy, but it is possible that this collection was indeed an SDHy. Case 1 similarly just described "subdural hematoma." Only case 2 described "reddish-brown CSF," a description close to that of classic cSDH.

In summary, we present 5 cases of cSDH in infant survivors of abusive head trauma, which illustrate important caveats regarding the $\mathrm{T} 2^{\star}$-GRE and SWI signal characteristics of chronic subdural hemorrhage. First, the fluid in $\mathrm{CSDH}$ is often isointense or hyperintense to CSF on T2*-GRE or SWI. Second, hemosiderin staining along the dural surfaces or neomembranes is commonly yet not universally present. When there is a paucity of hemosiderin, neomembranes can be very subtle. Because neomembranes are a key observation in diagnosing $\mathrm{cSDH}$, careful examination of all MR imaging sequences in multiple planes is necessary. These observations serve to further refine the described appearance of cSDH on T2*-GRE/SWI in infants with abusive head trauma and dispel any notion that chronic subdural hemorrhage must demonstrate hypointense fluid or hemosiderin. This refinement is particularly important in the setting of child abuse, given the critical medicolegal and forensic implications of accurate subdural collection description and dating.

Disclosures: Ulrich A. Rassner-UNRELATED: Consultancy: Elsevier (author); Payment for Lectures (including service on Speakers Bureaus): Siemens, Comments: speaker at Siemens 3T courses.

\section{REFERENCES}

1. Bradley WG Jr. MR appearance of hemorrhage in the brain. Radiology 1993;189:15-26 CrossRef Medline

2. Fobben ES, Grossman RI, Atlas SW, et al. MR characteristics of subdural hematomas and hygromas at 1.5 T. AJR Am J Roentgenol 1989; 153:589-95 CrossRef Medline 
3. Kleinman PK. Diagnostic Imaging of Child Abuse. Cambridge: University Press; 2015

4. Wittschieber D, Karger B, Niederstadt T, et al. Subdural hygromas in abusive head trauma: pathogenesis, diagnosis, and forensic implications. AJNR Am J Neuroradiol 2015;36:432-39 CrossRef Medline

5. Vezina G. Assessment of the nature and age of subdural collections in nonaccidental head injury with CT and MRI. Pediatr Radiol 2009; 39:586-90 CrossRef Medline

6. Hymel KP, Jenny C, Block RW. Intracranial hemorrhage and rebleeding in suspected victims of abusive head trauma: ad- dressing the forensic controversies. Child Maltreat 2002;7: 329-48 Medline

7. Weir B. The osmolality of subdural hematoma fluid. J Neurosurg 1971;34:528-33 CrossRef Medline

8. Hedlund GL. Subdural hemorrhage in abusive head trauma: imaging challenges and controversies. Am Osteopath Coll Radiol 2012;1:23-30

9. Bradford R, Choudhary AK, Dias MS. Serial neuroimaging in infants with abusive head trauma: timing abusive injuries. J Neurosurg Pediatr 2013;12:110-19 CrossRef Medline

10. Lee KS, Bae WK, Park YT, et al. The pathogenesis and fate of traumatic subdural hygroma. Br J Neurosurg 1994;8:551-58 Medline 